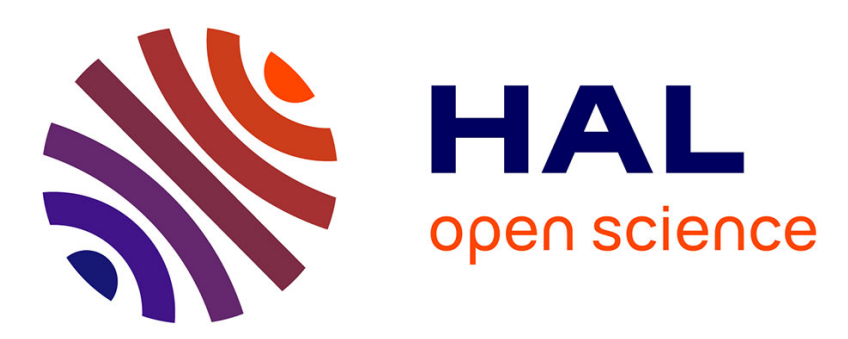

\title{
Modification of water retention and rheological properties of fresh state cement-based mortars by guar gum derivatives
}

\author{
Alexandre Govin, Marie-Claude Bartholin, Barbara Biasotti, Max Giudici, \\ Valentina Langella, Philippe Grosseau
}

\section{To cite this version:}

Alexandre Govin, Marie-Claude Bartholin, Barbara Biasotti, Max Giudici, Valentina Langella, et al.. Modification of water retention and rheological properties of fresh state cement-based mortars by guar gum derivatives. Construction and Building Materials, 2016, 122, pp.772 à 780 . 10.1016/j.conbuildmat.2016.06.125 . hal-01347733

\section{HAL Id: hal-01347733 \\ https://hal.science/hal-01347733}

Submitted on 25 Jul 2016

HAL is a multi-disciplinary open access archive for the deposit and dissemination of scientific research documents, whether they are published or not. The documents may come from teaching and research institutions in France or abroad, or from public or private research centers.
L'archive ouverte pluridisciplinaire HAL, est destinée au dépôt et à la diffusion de documents scientifiques de niveau recherche, publiés ou non, émanant des établissements d'enseignement et de recherche français ou étrangers, des laboratoires publics ou privés. 


\section{Modification of Water Retention and Rheological} properties of fresh state cement-based mortars by guar gum derivatives

Alexandre Govin ${ }^{a^{*}}$, Marie-Claude Bartholin ${ }^{\mathrm{a}}$, Barbara Biasotti ${ }^{\mathrm{b}}$, Max Giudici ${ }^{\mathrm{b}}$, Valentina Langella ${ }^{b}$, Philippe Grosseau ${ }^{a}$

a Ecole Nationale Supérieure des Mines, SPIN-EMSE, CNRS:UMR5307, LGF, F-42023 Saint-Etienne, France

${ }^{\mathrm{b}}$ Lamberti SpA, 21041 Albizzate, Italy

* Corresponding author: Tel: +33477420253

E-mail address: govin@emse.fr 


\begin{abstract}
The present study examines the influence of chemical composition and structure of guar gum derivatives on water retention capacity (WR) and rheological behavior of fresh state cementbased mortars. The investigation was also completed by adsorption isotherms. For this, original guar gum, three HydroxyPropyl Guars (HPG) and two hydrophobically modified HPGs were selected. The effect of the molar substitution $\left(\mathrm{MS}_{\mathrm{HP}}\right)$ and of hydrophobic substitution $\left(\mathrm{DS}_{\mathrm{AC}}\right)$ was investigated. The results highlight that chemical composition of HPGs has a remarkable effect on fresh state properties of mortars. The original guar gum does not impact on neither WR nor rheological behavior. Increasing $\mathrm{MS}_{\mathrm{HP}}$ leads to an improvement of the WR and the stability of mortars while the hydrophobic units further enhance WR and lead to a decrease in the yield stress and an increase in the resistance to the flow of admixed mortars.
\end{abstract}

Keywords: Admixture; Rheology; Water retention; Polysaccharide; Mortar; Guar derivatives.

\title{
HIGHLIGHTS
}

$>$ The influence of chemical composition and structure of guar gum derivatives on water retention capacity and rheological behavior of fresh mortars is examined.

$>$ The effect of the molar substitution $\left(\mathrm{MS}_{\mathrm{HP}}\right)$ and the degree of substitution of hydrophobic units $\left(\mathrm{DS}_{\mathrm{AC}}\right)$ was investigated.

Increasing $\mathrm{MS}_{\mathrm{HP}}$ leads to an improvement of the WR and the stability of mortars while the presence of alkyl chains promotes the WR and increases the resistance to the flow of admixed mortars. 


\section{Introduction}

Modern factory-made mortars are complex materials, in which several kinds of admixtures are added in order to obtain specific properties, from the fresh state to the hardened material. Indeed, since many years, concretes, mortars or cement grouts with high fluidity have been developed, since their use implies many economical and technical advantages. However, the use of highly flowable mixtures may lead to segregation or excessive bleeding and subsequently, durability issues. In order to overcome this problem by enhancing the sedimentation resistance while maintaining high fluidity, viscosity-enhancing admixtures (VEA) are frequently introduced within the formulation [1-4]. Among these admixtures, natural polysaccharides or their derivatives (such as welan gum, starch derivatives or cellulose ethers) are the most widely used. The incorporation of these VEAs in shotcrete or render mortar is useful to ensure sagging resistance for thick application on vertical support, and to allow sufficient fluidity for normal pumpability by supplying shear thinning rheological behavior [5]. Indeed, these admixtures provide, generally, high yield stress and apparent viscosity at low shear rate but low resistance to flow at high shear rate [6]. However, their mode of action is not fully understood, since results are sometimes contradictory and strongly dependent of the kind of binder, the polysaccharide nature and the molecular parameters of the admixture (such as molecular weight, nature and content of substitution groups) [7,8-15]. Water retention (WR) is another essential property of monolayer render at fresh state. Indeed, high water retention improves the cement hydration and limits the absorption of the mixing water by a substrate and thus provides good mechanical and adhesive properties to the mortar $[16,17]$. Among admixtures enhancing water retention capacity of the freshly-mixed mortars, cellulose ethers (CE) are the most widely used [11]. As in the case of the rheological 
properties, it appears that polymer molecular parameters, such as nature and content of substitution groups, and molecular weight, have a significant influence on WR $[11,18]$. The results demonstrated that the WR is strongly improved by the increase in the molecular weight of HydroxyEthylMethylCellulose (HEMC), HydroxyPropylMethylCellulose (HPMC) and HydroxyEthylCellulose (HEC). On the contrary, the molar substitution seems to have a lower impact on the water retention of admixed mortars. Nevertheless, the water retention is improved for low molar substitutions of the CE.

Despite a wide use of CE, HydroxyPropyl Guar (HPG) are now also well-established in the construction industry as water retention agents, as anti-sagging agents and rheology modifiers for mortars [19-23]. Moreover, HPGs are already widely used in various industrial fields, such as textile printing, hydraulic fracturing process, oil production or paper manufacturing, due to their thickening effect [24,25]. Consequently, since HPGs improve the two main properties of mortar, they appear as suitable admixtures to be used in render formulation. However, the study of their impact on mortars is still low, since Plank presented these natural polysaccharides as new promising class of water retaining agents in building materials [26]. Poinot et al. studied the impact of HPGs on WR and rheological properties of cement-based mortars [21,22]. However, since the main objective of the research was to elucidate the WR mechanism involved by CEs and HPGs, the formulation did not correspond to an industriallyused standard mixture. Indeed, high Liquid-to-Solid ratio and consequently high admixture dosages were used to be discriminant with respect to WR. Cappellari et al. also studied the effect of HPG and CE on WR and rheological properties of mortars [23]. However, the influence of the molecular parameters could not be studied since the only one commercial HPG was tested on a lime-based mortar. 
The aim of this study is to provide an understanding of the effect of chemical composition and structure of HPGs and its dosage on macroscopic properties of mortars. For this purpose, an original guar gum and five HPGs with specific chemical modifications, such as increase in $\mathrm{MS}_{\mathrm{HP}}$ or substitutions by hydrophobic units, were selected. The impact of admixtures on the water retention capacity and on the rheological behavior of mortars was investigated.

\section{Materials and methods}

\subsection{Mineral products}

Mineral products used in this study consist of Portland-composite cement (Holcim), lime (Holcim), calcium carbonate (Calcitec V60, Mineraria Sacilese S.p.A.) and two dolomites (Bombardieri noted Dolomite 1 and Leidi $0.1-0.4 \mathrm{~mm}$ noted Dolomite 2). The mineral composition of the commercial Portland-limestone cement used, CEM II/B-LL $32.5 \mathrm{R}$ according to the European standard EN 197-1 [27], is given in Table 1.

Table 1. Mineral composition (\%, weight) of the investigated cement determined by XRF and XRD-Rietveld refinement.

\begin{tabular}{|c|c|c|c|c|c|c|c|}
\hline \multicolumn{4}{|c|}{ Chemical composition $(\% \mathrm{wt})$} & \multicolumn{4}{|c|}{ Phase composition $(\% \mathrm{wt})$} \\
\hline Oxides & $\mathrm{XRF}$ & Oxides & $\mathrm{XRF}$ & Phases & $\begin{array}{c}\text { XRD } \\
\text { (Rietveld) }\end{array}$ & Phases & $\begin{array}{c}\text { XRD } \\
\text { (Rietveld) }\end{array}$ \\
\hline $\mathrm{CaO}$ & 57.87 & $\mathrm{SO}_{3}$ & 3.95 & $\mathrm{C}_{3} \mathrm{~S}$ & 54.3 & Calcite & 28.9 \\
\hline $\mathrm{SiO}_{2}$ & 12.31 & $\mathrm{Na}_{2} \mathrm{O}$ & 0.50 & $\mathrm{C}_{2} \mathrm{~S}$ & 3.5 & Gypsum & 3.0 \\
\hline $\mathrm{Al}_{2} \mathrm{O}_{3}$ & 2.63 & $\mathrm{~K}_{2} \mathrm{O}$ & 0.83 & $\mathrm{C}_{3} \mathrm{~A}$ & 4.7 & Quartz & 0.9 \\
\hline $\mathrm{MgO}$ & 1.19 & $\mathrm{TiO}_{2}$ & 0.16 & $\mathrm{C}_{4} \mathrm{AF}$ & 4.6 & Free $\mathrm{CaO}$ & 0.8 \\
\hline $\mathrm{Fe}_{2} \mathrm{O}_{3}$ & 2.03 & LOI & 13.7 & & & & \\
\hline
\end{tabular}

The phase composition was determined by Rietveld refinement method after XRD analysis (D5000, Siemens) and the oxide composition was quantified by means of X-ray fluorescence spectroscopy. The particle sizes were determined by means of laser diffractometry with dry 
powder disperser (Mastersizer 2000, Malvern). The median particle diameters by volume $\left(\mathrm{d}_{50} \%\right)$ are $630 \mu \mathrm{m}, 300 \mu \mathrm{m}, 20 \mu \mathrm{m}, 15 \mu \mathrm{m}$ and $5 \mu \mathrm{m}$ for the Dolomite 1, Dolomite 2, calcium carbonate, cement and lime, respectively. The particle size distribution and the specific surface area (determined by BET) are given in Fig. 1 and Table 2.

Table 2. Median particle diameters $\left(\mathrm{d}_{50}\right)$ and specific surface area of the mineral phases.

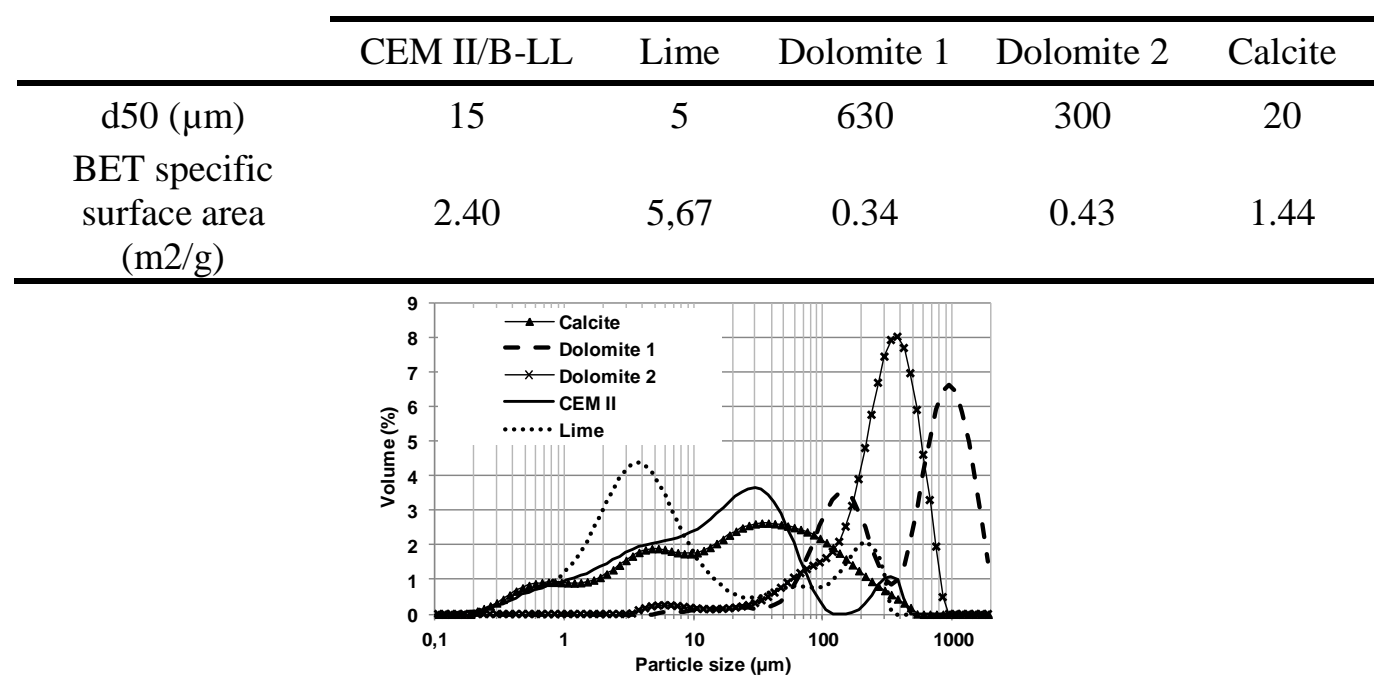

Fig. 1. Particle size distribution of raw materials constituting the mortar.

\subsection{Organic admixtures}

Guar gum is a high molecular weight, hydrophilic, non-ionic natural polysaccharide extracted from the endospermic seed of Cyamopsis tetragonolobus. Guar gum belongs to the large family of galactomannans and consists in a $\beta(1-4)$-linked D-mannopyranose backbone with random branchpoints of galactose via an $\alpha(1-6)$ linkage (Fig. 2 (a)). Hydroxypropyl guars (HPGs) are obtained from the original guar gum via an irreversible nucleophilic substitution, using propylene oxide in the presence of an alkaline catalyst (Fig. 2(b)). The manufacture of HPGs has the advantage of having a more reduced impact on the environment than cellulose derivatives. Indeed, guar gum is extracted by simple thermo-mechanical process, exhibits a higher chemical reactivity and is soluble in cold water thanks to its branched-chain structure 
with a lot of hydroxyl groups. Thus, the chemical modification of the original guar gum requires normal reaction conditions of temperature and pressure, does not generate large quantity of by-products, and requires minimal purification procedure [19].

(a)

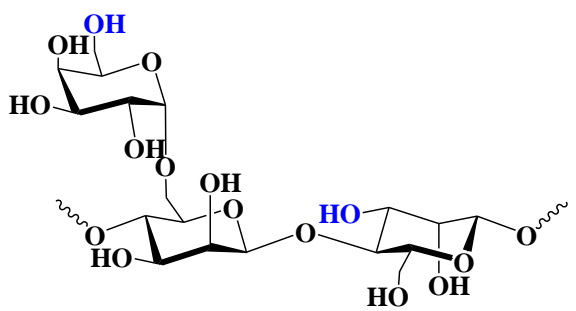

(b)

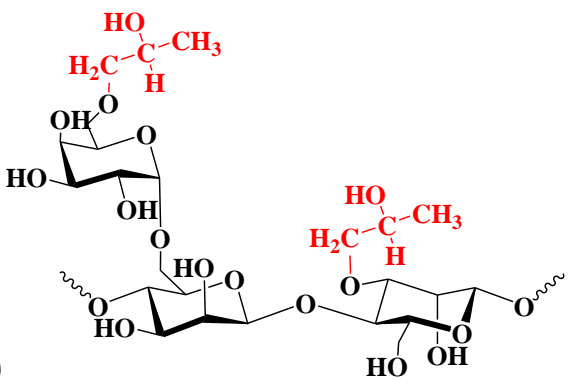

Fig. 2. Molecular structure of original guar gum (GG) (a) and HydroxyPropyl Guar (b).

In this paper, five HPGs and one original guar gum provided by Lamberti S.p.A were studied. They exhibit roughly the same molecular weight, around $2.10^{6} \mathrm{~g} \cdot \mathrm{mol}^{-1}$ since they are all from the same original guar gum (GG) [28].

Table 3. Qualitative description of the HPG used.

\begin{tabular}{cccc}
\hline & MS $_{\mathrm{HP}}$ & $\begin{array}{c}\text { Additional } \\
\text { substitution }\end{array}$ & $\mathrm{DS}_{\mathrm{AC}}$ \\
\hline GG & - & - & - \\
HPG 1 & Low & - & - \\
HPG 2 & Medium & - & - \\
HPG 3 & High & - & - \\
HPG 4 & High & Short alkyl chain & Higher DS than HPG 4 \\
HPG 5 & High & Short alkyl chain & Hon \\
\hline
\end{tabular}

Table 3 provides a qualitative description of the polymers used. The qualitative substitution degrees are provided by the manufacturers. The molar substitution ratio $\left(\mathrm{MS}_{\mathrm{HP}}\right)$ represents the number of hydroxypropyl units per hexose unit (mannose or galactose), which is, according to the manufacturer, less than 3 for the investigated HPGs. The degree of substitution $\left(\mathrm{DS}_{\mathrm{AC}}\right)$ represents the amount of alkyl chain per hexose unit. As in the case of the MS, the $\mathrm{DS}_{\mathrm{AC}}$ is less than 3 for the investigated HPGs. The only difference between HPGs 1, 2 and 3 is the 
molar substitution ratio, which increases from HPG 1 to HPG 3, while HPGs 4 and 5 exhibit an additional substitution (short alkyl chains) compared to HPG 3. The DS $\mathrm{AC}_{\text {of }}$ HPG 5 is higher than that of HPG 4.

\subsection{Preparation of fresh mortars}

Mortars were prepared according to the following mixture proportions: $12 \mathrm{wt} . \%$ of cement, 3 wt. $\%$ of lime, 18 wt. $\%$ of calcium carbonate, 43 wt.\% of dolomite Bombardieri and 24 wt.\% of dolomite Leidi. The admixtures were dry blended with the binders and fillers, and the dosages were expressed in percent by weight of solid ( $\%$ bwos). The polymer dosages used for the study are summarized in Table 4.

Table 4. Polymer dosages used in the mortar formulations. $x$ corresponds to the dosages tested for the Water Retention and adsorption measurements, o corresponds to the dosages used for the rheological measurements.

\begin{tabular}{cccccccccc} 
& \multicolumn{10}{c}{ Dosage (\%bwos) } \\
\cline { 2 - 9 } & 0.03 & 0.05 & 0.06 & 0.075 & 0.1 & 0.125 & 0.15 & 0.175 & 0.2 \\
\hline HPG 1 & & $\mathrm{x} / \mathrm{o}$ & & $\mathrm{x} / \mathrm{o}$ & $\mathrm{x} / \mathrm{o}$ & $\mathrm{x} / \mathrm{o}$ & $\mathrm{x} / \mathrm{o}$ & $\mathrm{x}$ & $\mathrm{x}$ \\
HPG 2 & & $\mathrm{x} / \mathrm{o}$ & & $\mathrm{x} / \mathrm{o}$ & $\mathrm{x} / \mathrm{o}$ & $\mathrm{x} / \mathrm{o}$ & $\mathrm{x} / \mathrm{o}$ & & \\
HPG 3 & & $\mathrm{x} / \mathrm{o}$ & $\mathrm{x}$ & $\mathrm{x} / \mathrm{o}$ & $\mathrm{x} / \mathrm{o}$ & $\mathrm{x} / \mathrm{o}$ & $\mathrm{x} / \mathrm{o}$ & & \\
HPG 4 & $\mathrm{x}$ & $\mathrm{x} / \mathrm{o}$ & $\mathrm{x}$ & $\mathrm{x} / \mathrm{o}$ & $\mathrm{x} / \mathrm{o}$ & $\mathrm{x} / \mathrm{o}$ & $\mathrm{x} / \mathrm{o}$ & & \\
HPG 5 & $\mathrm{x}$ & $\mathrm{x} / \mathrm{o}$ & $\mathrm{x}$ & $\mathrm{x} / \mathrm{o}$ & $\mathrm{x} / \mathrm{o}$ & $\mathrm{x} / \mathrm{o}$ & $\mathrm{x} / \mathrm{o}$ & & \\
GG & & $\mathrm{x} / \mathrm{o}$ & & & $\mathrm{x} / \mathrm{o}$ & $\mathrm{x} / \mathrm{o}$ & $\mathrm{x} / \mathrm{o}$ & $\mathrm{x}$ \\
\hline
\end{tabular}

The dry mixture was blended in a shaker (Turbula, Wab) for $10 \mathrm{~min}$. Deionized water was added in order to obtain a liquid-to-solid ratio $\mathrm{L} / \mathrm{S}=0.22$. The mixing was made in mixer (MIx40, CAD Instruments), in accordance to EN 196-1 [29]. The experimental strategy consisted in dividing the freshly mixed mortar into three parts in order to characterize several properties from the same mixing. A first part was used to study the rheological behavior of 
the mortar, the water retention measurements were performed on the second part and the third part of the freshly mixed mortar was centrifuged in order to determine the adsorption isotherms and the polymer concentration within the pore solution following a procedure described later.

All tests were carried out, at least, in triplicate and at a controlled temperature since water retention, rheological behavior of the mortar and adsorption isotherm are temperaturedependent. A control test was also performed with a mortar without admixture.

\subsection{Water retention measurements}

The water retention capacity of freshly-mixed mortar was assessed using the standard method described in ASTM C1506-09 [30]. The test had to be performed 15 min after mixing to measure the water loss of a mortar under vacuum. The standardized apparatus was submitted to a vacuum of $50 \mathrm{~mm}$ of mercury $\left(6.610^{3} \mathrm{~Pa}\right)$ for $15 \mathrm{~min}$. Then, the water retention capacity, WR, was calculated using the following equation:

$W R(\%)=\left(W_{0}-W_{1}\right) \times 100 / W_{0}$

where $W_{0}$ represents the initial mass of mixing water; $W_{l}$ is the loss of water mass after suction.

All the experiments were carried out at $23{ }^{\circ} \mathrm{C}$. Three classes of water retention (measured by ASTM method) of a fresh mortar can be specified according to the DTU 26.1 [31]. The first class (low water retention category) contains mortars that exhibit a water retention lower than $86 \%$. The second class (intermediate) corresponds to values ranging from $86 \%$ to $94 \%$. The last one (strong) is defined by water retention higher than $94 \%$, corresponding to the required values in the field of rendering application. 


\subsection{Rheological behavior}

The rheological measurements were performed with a Rheometer MCR 302 (Anton-Paar), thermostated at $20{ }^{\circ} \mathrm{C}$. The rheological properties of fresh mortars were investigated with vane-cylinder geometry since this system is suitable for granular pastes like mortars $[13,32]$. The gap thickness, distance between the periphery of the vane tool and the outer cylinder, was set at $8.5 \mathrm{~mm}$, in order to be less sensitive to the heterogeneity of the mortar. Using a Couette analogy, the shear stress and shear rate were calculated from the torque and the applied rotational velocity respectively, after calibration with glycerol [33]. The mortar was introduced into the measurement system at the end of the mixing cycle and was then held at rest. At $10 \mathrm{~min}$, the mortar was pre-sheared for $30 \mathrm{~s}$ at $100 \mathrm{~s}^{-1}$ in order to re-homogenize the sample and to eliminate its shear history because of potential thixotropic character of cementitious materials $[34,35]$. After a period of rest of $5 \mathrm{~min}$, the rheological measurements were started. The imposed shear rate was 16 decreasing steps from 300 to $0.06 \mathrm{~s}^{-1}$. The measuring time was adjusted for each shear rate, in order to obtain a steady state. The samples were systematically submitted to high shear rate $\left(100 \mathrm{~s}^{-1}\right)$ for $30 \mathrm{~s}$ before each imposed shear rate in order to resuspend particles of mortar within the mortar mixtures.

The shear stress $(\tau)$ was expressed as a function of the shear rate $(\dot{\gamma})$ and the Herschel-Bulkley (HB) model was applied to fit the experimental data and used to describe mortars rheological behavior [36]:

$$
\tau=\tau_{0}+K \dot{\gamma}^{n}
$$

where $\tau_{0}$ corresponds to the yield stress, $K$ the consistency coefficient and $n$ the fluidity index which characterizes shear-thinning behavior of mortar. 


\subsection{Adsorption curves of HPGs on binder}

The adsorption isotherms were determined using the depletion method. The non-adsorbed polymer remaining within the pore solution was quantified by means of Total Organic Carbon (TOC) measurements. Prior to the analysis, the pore solution was extracted from the mortar by means of two centrifugation steps, 15 min after contact between binder and water. The first step consisted in the centrifugation of around $150 \mathrm{~g}$ of mortar at $5000 \mathrm{rpm}$ for $5 \mathrm{~min}$. The supernatant was, afterward, centrifuged again at $14500 \mathrm{rpm}$ for $10 \mathrm{~min}$ in order to avoid the presence of mineral particles within the solution. The supernatant was diluted with hydrochloric acid solution at $0.1 \mathrm{~mol} . \mathrm{L}^{-1}$. The TOC was determined by combustion at $850{ }^{\circ} \mathrm{C}$ with a Vario-TOC Cube (Elementar). The adsorbed amount of polysaccharides was calculated from the difference of TOC content of the HPG reference solution and the TOC content of the supernatant.

\subsection{Hydrodynamic radius measurement using dynamic light scattering}

The dynamic light scattering (DLS) measurements were performed using a Zetasizer Nano ZS (Malvern Instrument). The wavelength of the incident light was $633 \mathrm{~nm}$ and the scattered light was detected at $173^{\circ}$. The admixtures were dissolved in lime solution $(20 \mathrm{mM})$ during $24 \mathrm{~h}$ under magnetic stirring. The concentration in polymer was fixed to $0.2 \mathrm{~g} . \mathrm{L}^{-1}$. The solutions were filtered through a $0.45 \mu \mathrm{m}$ filter in order to remove dust before testing.

\subsection{Dissolution kinetics of the admixture after the mixing}

The dissolution process of the admixtures was monitored by TOC measurements. However, all the solid phases were substituted by normalized sand [29]. This avoids evolution of the system due to the hydration process, it limits potential adsorption of polymer on cement phases [37] and size distribution of the mineral phases is close to that of the binder. The 
admixture was first added to the sand and the dry mixture was blended in a shaker (Turbula, Wab) for $10 \mathrm{~min}$. The amount of polymer was the same as for a dosage of $0.05 \%$ bwos in mortars. Then, a $20 \mathrm{mM}$ lime solution was added in order to keep high $\mathrm{pH}$ and to replace the pore solution (simplified model). The liquid-to-solid ratio was maintained at 0.22 (similar than mortars). Finally the mixture was mixed for 4 min according to EN 196-1 standard [29]. At 5, 15 and 30 min (i.e. 1, 11 and 26 min after the end of the mixing, respectively), a part of the mixture was centrifuged and the amount of polymer was determined by TOC measurements with same experimental procedure than in the case of the adsorption quantification.

\section{Experimental Results}

\subsection{Impact of HPGs on the water retention property of fresh mortars}

Fig. 3 represents the evolution of the water retention capacity of fresh admixed mortars, according to the polymer dosage. The non-admixed mortar exhibits a low water retention capacity of about $71.9 \% \pm 0.7 \%$. Then, the water retention increases with the use of HPGs and with increasing polymer dosage [21,23], until reaching a plateau with very high WR values (>98\%). In the range of polymer dosage studied, the WR values reached for HPG 1 to HPG 5, are greater than 94\% and therefore belong to the strong WR class. However, according to the admixture, this WR value is reached for different admixture dosages. Indeed, HPG 1 is able to provide a strong WR to the mortar for dosages ranging between 0.175 and $0.2 \%$ bwos, corresponding approximately to twice and three times the required dosage with HPG 3 and HPGs 4 and 5, respectively. One can also clearly notice the very limited impact of the original guar gum regardless of the dosage tested, since the WR provided by the gum is lower than $75 \%$ and is very close to that of the non-admixed mortar $(\approx 72 \%)$. These results 
suggest that the substitution of the hydroxyl units from original guar gum by hydroxypropyl units allows us to increase the WR of mortars. Furthermore, the increase in the $\mathrm{MS}_{\mathrm{HP}}$ (from HPG 1 to HPG 3) improves the WR capacity of mortar, since HPG 3 provides the higher WR despite lower dosage, followed by HPG 2 and then by HPG 1. These results are consistent with those obtained by Poinot et al. [21].

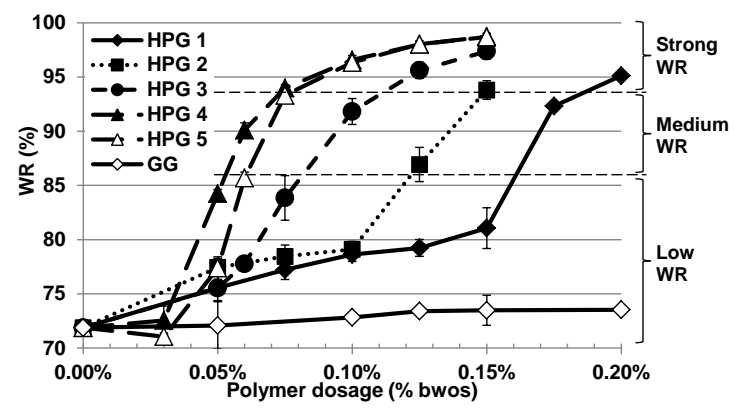

Fig. 3. Impact of polymer dosage on water retention capacity of fresh admixed mortars.

The results highlight also the positive impact of additional alkyl chain on WR of mortars. Indeed, the water retention is strongly improved by the use of hydrophobically modified HPGs (HPG 4 and HPG 5) compared to HPG 3, even at low dosages. In the range of the dosage studied, the highest WR were obtained with these both hydrophobically modified HPGs and the strong WR class was reached for the lowest polymer dosages.

Moreover, as far as the shape of WR curves is concerned, an abrupt change in slope can be noticed for mortars admixed with HPG 1 to HPG 5. The origin of this point will be discussed later. The change in slope occurs for a decreasing polymer dosage from HPG 1 to HPG 3 and from HPG 3 to HPG 4 and HPG 5.

\subsection{Adsorption curves of HPGs on binder}

From the TOC measurements, the polymer concentration within the extracted pore solution was determined. Fig. 4 shows the evolution of this concentration as a function of the polymer 
dosage introduced. Excepted the original guar gum (GG), the amount of non-adsorbed polymer increases with increasing polymer dosage. The concentration of the GG within the pore solution is the lowest of all the tested admixtures and corresponds to less than $1.5 \%$ of the introduced dosage. The case of GG will be discussed later (section 4). The presence of hydroxypropyl substitutions on the guar leads to an increase in the polymer concentration since the amount of GG within the pore solution is around 24 and 43 times lower than HPG 1 and HPG 3, respectively. According to the admixture, the concentration rises following this order: GG < HPG $1<$ HPG $2<$ HPG $5<$ HPG $4<$ HPG 3.

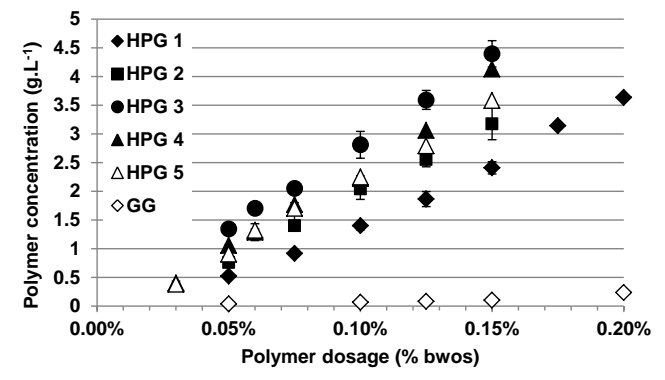

Fig. 4. Concentration of HPGs and original guar gum (GG) within the extracted mortar pore solution, expressed as function of the introduced polymer dosage.

In order to check if the non-adsorbed polymer coils were not trapped into the porosity of the fresh mortar during extraction of the pore solution, adsorption measurements were carried out with a very large excess of water $(\mathrm{L} / \mathrm{S}=2)$. In these conditions, due to the large excess of water, the admixture is not trapped into the porosity of the paste. Consequently, the amount of missing polymer can be ascribed to its adsorption onto the surface of solid particles. The concentration in admixture was determined by TOC measurements using the protocol described in section 2.6. This protocol was applied to HPG 1 and HPG 4, with a dosage fixed at $0.1 \%$ bwos. For HPG 1 , the amount of retained polymer was equal to $0.69 \mathrm{mg} / \mathrm{g}$ and 0.70 $\mathrm{mg} / \mathrm{g}$ for the L/S ratio of 0.22 and 2, respectively. For the same L/S ratios, the amounts of 
HPG 4 retained were equal to $0.51 \mathrm{mg} / \mathrm{g}(\mathrm{L} / \mathrm{S}=0.22)$ and $0.45 \mathrm{mg} / \mathrm{g}(\mathrm{L} / \mathrm{S}=2)$. This results show that whatever the liquid-to-solid ratios tested ( 0.22 or 2$)$, the amount of missing polymer is quite similar, confirming that hydroxypropyl guars are mainly adsorbed onto the surface of solid particles rather than entrapped into the porosity of the paste. The results are consistent with the large amount of polar groups on HPGs, such as hydroxyl groups, leading to interactions between the admixtures and the polar cement phases [38, 39].

Fig. 5 shows the adsorption isotherms of the admixtures on Portland based-mortars, as a function of the real polymer concentration in the pore solution (data extracted from Fig. 4).
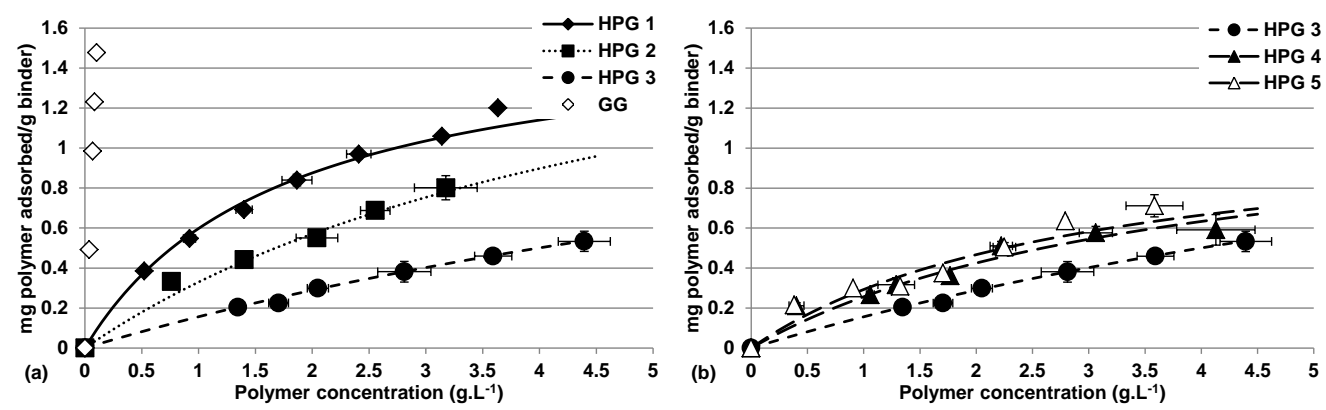

Fig. 5. Adsorption isotherms of HPG 1 to HPG 3 (a), and HPG 3 to HPG 5 (b) on binder, expressed as function of the polymer concentration in the pore solution obtained from TOC measurements. The data were fitted with Langmuir equation excepted the original guar gum.

Excepted GG, the experimental data were successfully fitted using a Langmuir model. In the range of polymer dosage tested in this study, no plateau was reached whatever the HPG. This suggests that either all the sites were not occupied or no equilibrium between adsorption and desorption reactions was achieved [40]. However, the strict linearity seems to be overcome (reduction in slopes), meaning that the amount of available adsorption sites starts to decrease. The increase in hydroxypropyl substitutions on the guar (increasing $\mathrm{MS}_{\mathrm{HP}}$ ) leads to a decrease in the adsorption of the admixture onto the solid surfaces. Indeed, the adsorption is reduced, respectively, by $18 \%$ and $45 \%$ for HPG 2 and HPG 3, with respect to HPG 1 (Fig. 5(a)). This 
tendency is consistent with previous studies conducted on HPGs and cellulose ethers (CE) in cementitious materials $[15,20]$.

Fig. 5(b) highlights the effect of the additional alkyl chain on the adsorption. It appears that the hydrophobic side chains slightly intensify the adsorption of the hydrophobically modified HPGs onto the surface of grains with respect to HPG 3 . However, the adsorption of HPG 4 and HPG 5 is still lower than that of HPG 2. The effect of the $\mathrm{DS}_{\mathrm{AC}}$ appears as negligible since the experimental data from HPG 4 and HPG 5 are superimposed.

\subsection{Impact of HPGs on the rheological properties of fresh mortars}

Prior to rheological measurements, the thixotropy of the mortars was evaluated. The results (not shown here) suggest that thixotropy does not affect the rheological measurements. Indeed, in the range of tested shear rates and thanks to the experimental procedure, the shear stress obtained by the increasing or decreasing shear rate ramps are superimposed which justifies the choice to consider only the decreasing ramps for all the rheological study.

Fig. 6 shows the evolution of the yield stress, extracted from Herschel-Bulkley model, for all the studied mortars with and without admixture. The mortar without admixture exhibits a yield stress value of around $45 \mathrm{~Pa}$. From the presented results, three different classes of HPG, inducing different evolutions of the yield stress with the polymer dosage, can be highlighted for admixed mortars. The first category is only composed of the original guar gum (GG), which induces a low and quasi linear decrease in the yield stress of mortar when admixture dosage increases. On the contrary, HPGs 1, 2 and 3 lead to a continuous rise of the yield stress of mortars from 50-60 Pa to around $120 \mathrm{~Pa}$ with the increase in the HPG dosage from $0.05 \%$ to $0.15 \%$. Finally, HPG 4 and HPG 5 constitute the third class of admixture. The use of these admixtures leads to an improvement of the yield stress compared to the non-admixed 
mortar, whatever the dosages tested in the study. However, the improvement is not proportional to the admixture dosage. Indeed, our first dosage $(0.05 \%$ bwos $)$ leads to an increase in the yield stress. Beyond this dosage, increasing the dosage provides a low decrease in the yield stress, before reaching a plateau. The value of the yield stress reached on the plateau $(\approx 60 \mathrm{MPa})$ is still higher than that of the mortar without admixture.
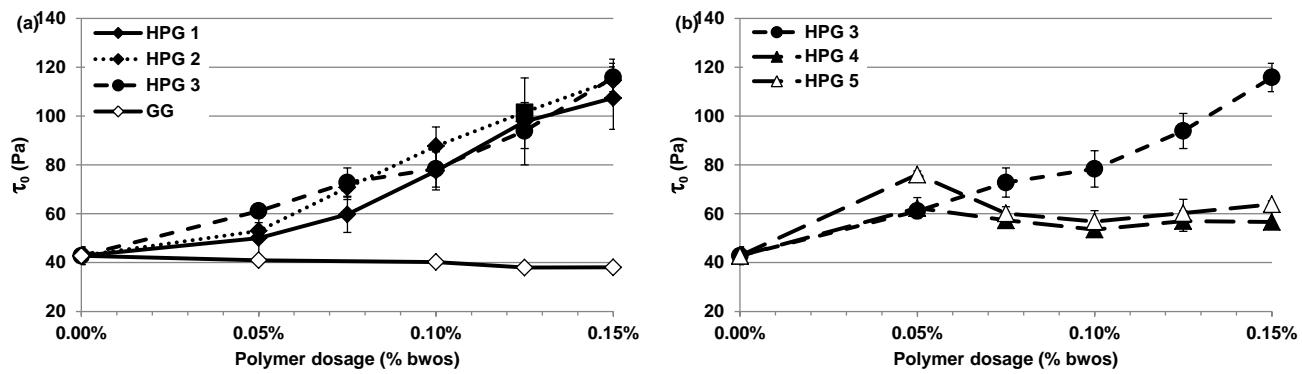

Fig. 6. Impact of polymer dosage on yield stress of fresh admixed mortars (HPG 1 to HPG 3 and original guar gum (GG) (a) and HPG 3 to HPG 5 (b)).

The evolution of the consistency coefficient ( $K$ from Herschel-Bulkley equation) during the increase of polymer dosage is presented Fig. 7.
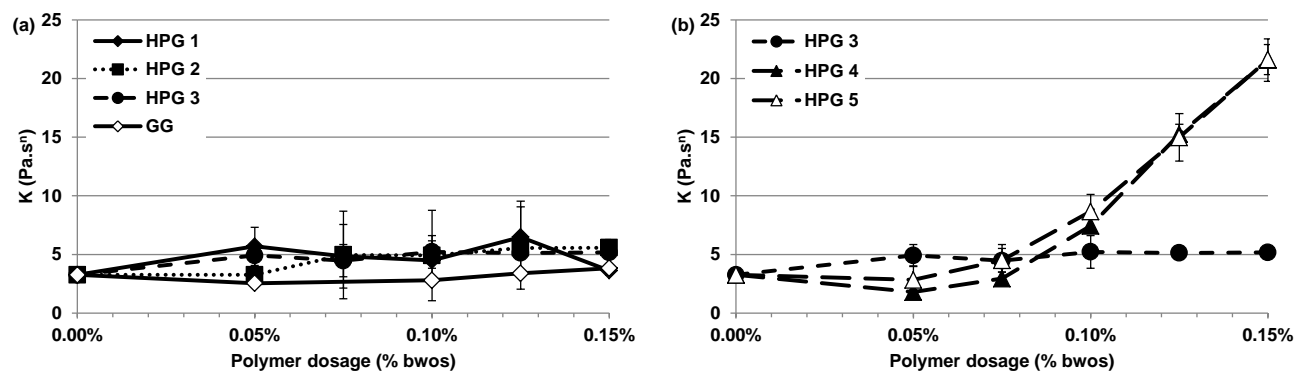

Fig. 7. Impact of polymer dosage on consistency coefficient of fresh admixed mortars (HPG 1 to HPG 3 and original guar gum (GG) (a) and HPG 3 to HPG 5 (b)).

As in the case of the yield stress, the results suggest that the admixtures can be divided into three classes. The first class is only composed of the original guar gum which provides a very low or negligible modification of the consistency coefficient with increasing polymer dosage 
compared to non-admixed mortar. HPG 1 to HPG 3, constituting the second group, induce first an increase followed by a plateau in the consistency coefficient $\left(\approx 5 \mathrm{~Pa} \cdot \mathrm{s}^{\mathrm{n}}\right)$. Finally, HPG 4 and HPG 5 lead to a continuous increase, up to $22 \mathrm{~Pa}^{\mathrm{n}} \mathrm{s}^{\mathrm{n}}$, in the consistency coefficient of admixed mortars.

Fig. 8 shows the evolution of the fluidity index $(n)$ versus the polymer dosage for all the studied mortars.
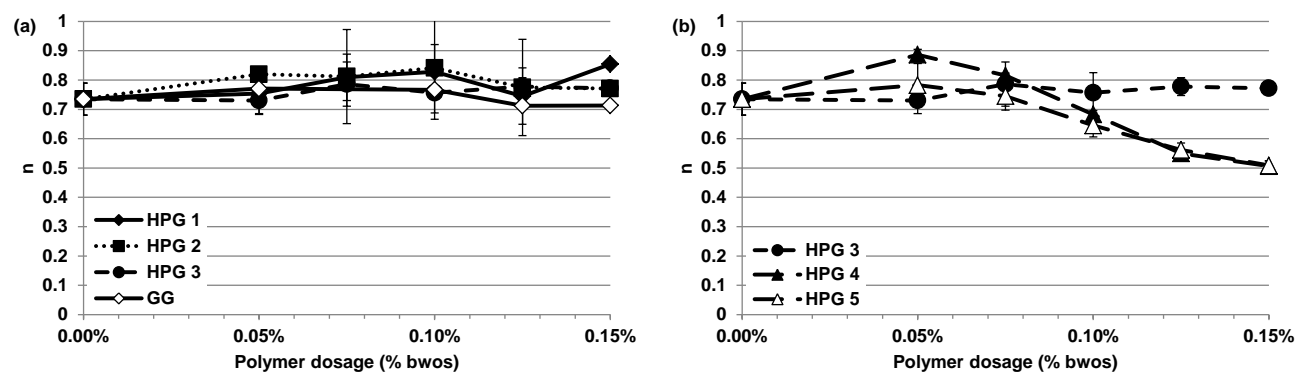

Fig. 8. Impact of polymer dosage on fluidity index of fresh admixed mortars (HPG 1 to HPG 3 and original guar gum (GG) (a) and HPG 3 to HPG 5 (b)).

Whatever the mortars (non-admixed and admixed), the values of the fluidity index are lower than 1 , meaning that they are all shear thinning. Due to the high standard deviation, the value of the fluidity index of mortars admixed with HPG 1 to HPG 3 and GG seem to be unchanged as the dosage of HPGs increase. However, HPGs 4 and HPG 5 leads to a low increase followed by a continuous decrease in the fluidity index until reaching values around 0.5 . It means that the shear thinning behavior of mortars becomes more and more pronounced.

\subsection{Hydrodynamic radius measurements using dynamic light scattering}

The hydrodynamic radii of the original guar gum (GG) and three HPGs (1, 3 and 4) were determined in lime solution (20mM) (Fig. 9). 


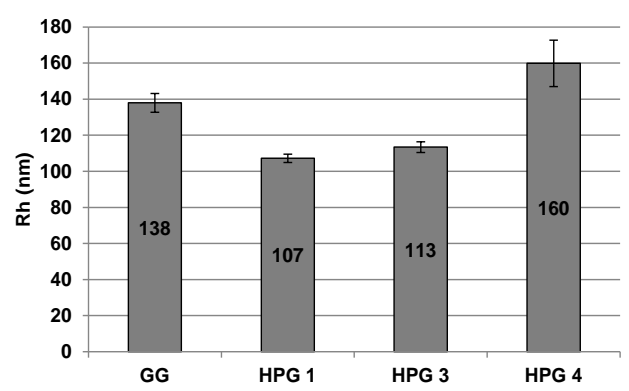

Fig. 9. Hydrodynamic radius of HPGs 1, 3, 4 and original guar gum (GG) in lime solution (20 $\mathrm{mM})$, (polymer concentration was fixed at $0.2 \mathrm{~g} \cdot \mathrm{L}^{-1}$ ).

Guar gum exhibits a radius of $138 \pm 5 \mathrm{~nm}$. The hydrodynamic radius decreases when hydroxypropyl substitutions are grafted on the original guar gum (107 $\pm 2 \mathrm{~nm}$ for HPG 1). The increase in the molar substitution leads to a slight increase in the hydrodynamic radius of the HPG (113 $\pm 3 \mathrm{~nm}$ for HPG 3). The same tendency was previously described by Cheng et al. by studying the effect of hydroxypropyl molar substitution on the molecular volume of guar gum [41]. According to the authors, this trend is the result of two antagonist effects induced by the hydroxypropyl groups grafted on the chain: the reduction of the intermolecular attractions which leads to a decrease in the hydrodynamic radius, and an increase in the local rigidity of the chain, which, as a result, increases the hydrodynamic radius [41]. Contrary to carboxymethylpullulans [42], the grafting of alkyl chains on HPG (HPG 4) leads to a higher hydrodynamic radius $(160 \pm 13 \mathrm{~nm})$ than that of HPGs.

\section{Discussion}

The effect of the original guar gum (GG) on all the studied macroscopic properties is negligible. From the Water Retention point of view, this result is coherent with the very low concentration of the GG within the pore solution (Fig. 4). Indeed, since the concentration in 
GG is close to $1.5 \%$ of the initial amount of polymer, very few molecules are in the pore solution. The composition of pore solution is thus very close to that of the non-admixed mortar, leading to similar WR. Indeed, WR is governed by the ability of the admixture to form aggregates and to plug the porous network of a thin polysaccharide-enriched filter cake [21], which can be, as first approximation, linked to the concentration.

Two assumptions were proposed to explain the very low content of the original guar gum in the pore solution: either the polymer is adsorbed onto the solid particles and/or trapped during hydration process [43]; or the dissolution of admixtures during the mixing is low. In order to answer, the dissolution kinetics of GG and HPGs 1, 3 and 4 were monitored in a simplified system composed only of normalized sand, admixture and lime solution (20 mM).
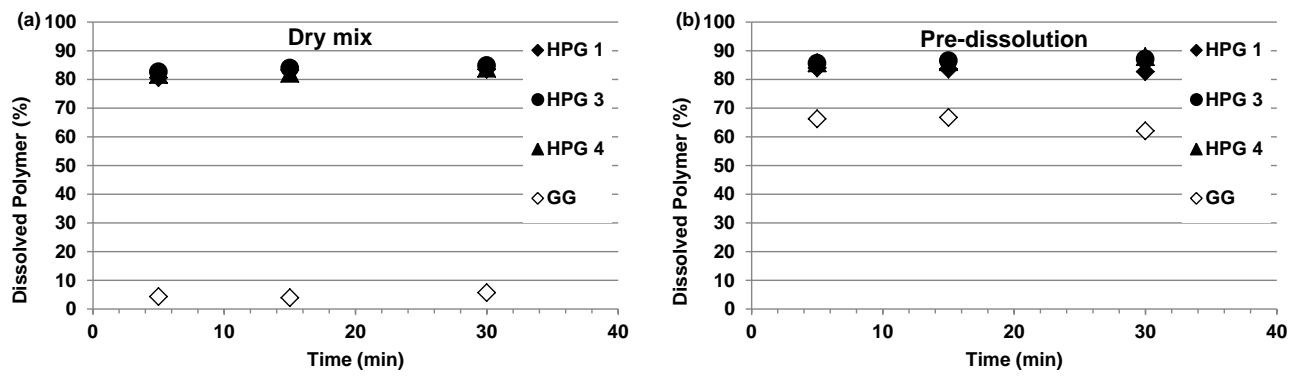

Fig. 10. Dissolution kinetics of HPGs 1,3 and 4, and original guar gum (GG) at 5, 15 and 30 minutes after the beginning of mixing with sand, in Dry mix (a) and after a pre-dissolution of the admixtures for $24 \mathrm{~h}$ in lime solution $(20 \mathrm{mM})(\mathrm{b})$.

The results highlight that the dissolution in the mixer is fast and similar for the three HPGs (Fig. 10(a)). Indeed, the data obtained for HPGs 1, 3 and 4 are superimposed. Moreover, more than $80 \%$ of the polymer is dissolved within the first $5 \mathrm{~min}$. Afterward the dissolution process up to 30 min leads to an increase in the dissolved polymer of only $3 \%$. The strong shear forces induced in the mixer and the friction with the granular media promote the dissolution of the HPGs. However, less than $6 \%$ of the original guar gum is released within the solution 
during the first $30 \mathrm{~min}$. These results suggest that GG was not trapped during the hydration process and/or adsorbed onto the surface of the cement.

In order to confirm the second hypothesis proposed (low dissolution of the original guar gum), the same experiments were conducted after a pre-dissolution of the admixtures. The admixture was added to the lime solution and stirred for $24 \mathrm{~h}$ prior its use. In these conditions, the polymer was totally dissolved in the solution before being introduced in the mixer.

The results show that the amount of HPGs 1, 3 and 4 detected in the solution after mixing with sand, is very slightly increased compared to dry-mixing experiments, confirming the fast dissolution of these admixtures (Fig. 10(b)). However, since the amount of polymer is not equal to $100 \%$ of the introduced amount, the adsorption onto surface of sand seems to occur (about 15\%). Contrary, the amounts of GG in the solution strongly increase since they reach values between $60 \%$ and $70 \%$ of the introduced amount of polymer, validating the slow dissolution kinetics in the dry mix. However, the original guar gum is strongly adsorbed onto sand surfaces since more than $30 \%$ of the initial amount of polymer is still missing. This result is in accordance with the adsorption of guar gum onto quartz previously shown by $\mathrm{Ma}$ and Pawlik [44].

Concerning the hydroxypropyl guar, the results of WR experiments are consistent with those of previous studies performed with HPGs or CEs and with the proposed mechanism $[21,45,46]$. Indeed, the WR of admixed mortars is mainly governed by the ability of polysaccharidic admixtures to form a hydrocolloidal associated polymer molecules network and to induce overlapping of polymer coils within the pore solution $[21,45,46]$. When the concentration of polymer increases in solution, the isolated polymer coils, existing at low polymer concentration, begin to come into contact with one another. This concentration is defined as the coil-overlap concentration (noted $C^{*}$ ). Above this critical concentration, the 
polysaccharide aggregates stop the water flow by plugging the porous network of a thin polysaccharide-enriched filter cake at the interface mortar-substrate resulting in a sudden and sharp rise in WR curves [21]. As previously mentioned, the abrupt change in slope is reached for a decreasing polymer dosage from HPG 1 to HPG 3. The only difference between these HPGs is the increasing substitution degree. According to literature, the increase in $\mathrm{MS}_{\mathrm{HP}}$ does not lead to a change in the $C^{*}[47]$. However, the increasing substitution degree leads to a decrease in adsorption of polymer (Fig. 5) and hence to a rise in polymer concentration in the pore solution (Fig. 4). Consequently, the coil overlapping occurs at lower dosage. The results highlight furthermore the positive impact of additional alkyl chain on WR.

The presence of additional alkyl chains (HPG 4 and HPG 5), despite a slight rise in adsorption compared to HPG 3, leads to the formation of polymer aggregates at lower polymer dosage. Indeed, the interconnection between alkyl chains creates intramolecular and intermolecular interactions through specific hydrophobic interactions which cause a decrease in the coiloverlapping concentration $[41,42,48]$. Consequently, the abrupt change in slope is reached for HPG 4 and HPG 5 at lower polymer dosage than HPG 3. However, an increase in the $\mathrm{DS}_{\mathrm{AC}}$ (from HPG 4 to HPG 5) can lead to a conversion of some intermolecular associations to intramolecular associations and hence an increase in the polymer dosage necessary to reach coil overlap [47].

The rheological results (Fig. 6, Fig. 7 and Fig. 8) highlight that HPG 1 to HPG 3, HPG 4 to HPG 5 and GG behave quite differently. Indeed, HPGs 1, 2 and 3 lead to a continuous increase in the yield stress, while HPG 4 and HPG 5 modify mainly the consistency coefficient and the fluidity index. This means that HPG 1 to HPG 3 increase the stability of mortars while HPG 4 and HPG 5 increase the resistance to the flow of admixed mortars. 
HPG 1 to HPG 3 affect the rheological behavior of the admixed mortars in the same way, i.e. an increase in the yield stress, a low increase followed by a plateau in the consistency coefficient and a negligible modification of the fluidity index when the polymer dosage rises. These results are in agreement with several papers reporting that an increase in VEA dosage leads to a rise in the yield stress $[2,4,14]$. However, Poinot et al., using the same type of HPGs, observed a decrease in the yield stress when the admixture dosage increases [22]. The different rheological behavior may be explained by the different mineral composition and the higher L/S ratio (0.3) of the mortars which are known to strongly affect the rheological properties [7].

Fig. 5 shows that HPGs adsorb onto particles constituting the mortar. Prima facie, this adsorption could be responsible for the increase in the yield stress because of bridging flocculation $[6,15,49]$. The bridging was highlighted thanks to rheological measurements, by applying to the mortar (non-admixed and admixed with $0.1 \%$ of HPG 3 and HPG 4) a constant shear rate of $0.05 \mathrm{~s}^{-1}$ during $180 \mathrm{~s}$. This was performed after the pre-sheared step and the period of rest previously described in the section 2.5 . The rheometer, the mobile and the cell were also the same. Fig. 11 shows the shear stress as a function of shear strain, for nonadmixed mortar and for mortar admixed with HPG 3 and HPG 4.

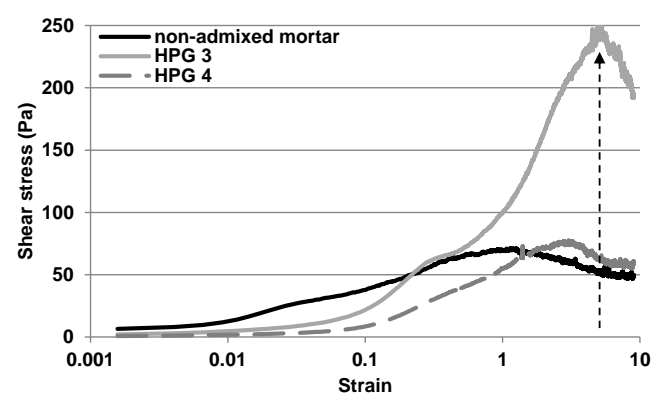

Fig. 11. Flow onset measurements for the non-admixed mortar and for mortars containing $0.1 \%$ of HPG 3 and 4 . 
A stress peak can be noticed, corresponding to "static yield stress. This stress is characteristic of the strength of the percolated network of cement particles $[15,35]$ and linked to a critical strain necessary to initiate the flow. It appears clearly that HPG 3 strongly increases the yield stress, which is consistent with the results shown in Fig. 6(a). The critical strain linked to this yield stress also increases until reaching a couple of $100 \%$. Brumaud et al. published similar results by studying cellulose ethers [15]. According to the authors, the increase in the critical strain is the result of the partial stretch of polymer chains beforehand adsorbed onto two cement particles (corresponding to bridging flocculation). This theory is compatible with the polymer chain length of our HPGs which can be estimated between 5 and $10 \mu \mathrm{m}$ (thanks to $\mathrm{Rh}, \mathrm{Mw}$ and DP). However, despite a strong drop of the adsorption (45\%) with the increase in the $\mathrm{MS}_{\mathrm{HP}}$ from HPG 1 to HPG 3, the yield stress also increases. This suggests that the nonadsorbed polymer may be responsible for the yield stress increase. The potential loss of bridging can be compensated by an increase in the pore solution viscosity (not shown here but already demonstrated in [22]) induced by the rise in the polymer concentration (Fig. 4) and/or by the depletion flocculation induced by the non-adsorbed coils [50].

Moreover, the presence of HPG coils within the pore solution leads to an increase in the consistency coefficient $(K)$ compared to non-admixed mortars. However, the expected increase in $K$ due to the rise of pore solution viscosity with the polymer dosage may be compensated by steric hindrance, leading to a plateau for $K$.

The rheological behavior of the admixed mortar with GG is very close to that of the nonadmixed mortar. This result is consistent with the very low amount of polymer dissolved due to the low dissolution kinetics. However, when the admixture dosage increases, the amount of polymer molecules available and the adsorption onto solid surfaces also increases. This can lead to an increase in the steric hindrance and in the dispersion and lubrication effects, leading 
to a low but continuous decrease in the yield stress. For dosages higher than $0.1 \%$ bwos, the concentration in polymer coils into the pore solution begins to slightly increase (Fig. 4), leading to the beginning of the increase in the consistency coefficient and of the decrease in the fluidity index.

The additional alkyl chain also modifies the rheological properties of mortars. Contrary to HPG 3, hydrophobically modified HPGs (HPG 4 and HPG 5) lead to a strong and continuous increase in the consistency coefficient and a decrease in the fluidity index. These results highlight that mortars become more and more shear-thinning since the fluidity index decreases from 0.8 to 0.5 . This rheological behavior gets more pronounced as the HPG dosage increases. These results are consistent with the rise of hydrodynamic radius and the promotion of the entanglement induced by the additional alkyl chains leading to the overlapping of polymer coils at lower dosage $(0.05 \%$ in this study). The enhancement of entanglement by additional alkyl chains was already established in a previous paper [21]. Above this dosage, entanglement of polymer coils induces a shear thinning behavior to the solution. The shear thinning behavior of the solution, and thus of the mortars, amplify with the increasing polymer dosage.

The yield stress is also impacted by the additional alkyl chain. Indeed, $\tau_{0}$, of mortar admixed with HPG 4 and HPG 5, increases for a dosage equal to $0.05 \%$ bwos then slowly decreases for dosages ranging from $0.05 \%$ to $0.075 \%$ bwos, before reaching a plateau for higher dosages ( $\tau_{0}$ reached is still slightly higher than that of the non-admixed mortar). This trend could be the result of antagonist effects such as the increase in pore solution viscosity with the dosage (not shown here but already demonstrated in [22]), which should promote the yield stress, and the increase both in adsorption onto the surface of particles (Fig. 5(b)) and in hydrodynamic radius (Fig. 9). The two last phenomenon lead to steric hindrance which 
implies a prevention of direct contacts between particles. Moreover, Fig. 11 shows that the static yield stress is reduced by HPG 4 in comparison to HPG 3, confirming the results of Fig. 6(b). This, also, confirms the reduction of the bridging ability of hydrophobically modified HPGs since the critical strain decreases. All these points could lead to a decrease in the yield stress.

\section{Conclusions}

This study was dedicated to the effect of several guar gum derivatives on water retention property and rheological behavior of mortars. Based upon the results, it was found that the original guar gum was very weakly dissolved, leading to a negligible modification of WR and rheological behavior with respect to the non-admixed mortar. Depending of the chemical structure of HPGs, it is possible to promote the water retention according to two different ways. First, by increasing the $\mathrm{MS}_{\mathrm{HP}}$ of HPGs, the amount of adsorbed polymer drops, which leads to an increase in the HPG concentration within the pore solution and therefore to lower HPG dosage necessary to reach coil overlapping. Second, by enhancing overlapping, the hydrophobically modified HPGs improve the effectiveness of WR agent at low dosage. HPGs also modify the rheological behavior of the mortars. As in the case of WR, the hydrophobic characteristic of HPGs is the preponderant parameter. Indeed, it was shown that additional alkyl chain mainly leads to a more shear thinning behavior of the mortar and to a rise in the consistency coefficient, while classical HPGs strongly increases the yield stress. 
Construction and Building Materials 122 (2016) 772-780

\section{References}

[1] S. Rols, J. Ambroise, J. Péra, Effects of different viscosity agents on the properties of selfleveling concrete, Cem. Concr. Res. 29 (2) (1999) 261-266.

[2] K.H. Khayat, A. Yahia, Effect of welan gum-high-range water reducer combinations on rheology of cement grout, ACI Mater. J. 94 (5) (1997) 365-372.

[3] M. Lachemi, K.M.A. Hossain, V. Lambros, P.-C. Nkinamubanzi, N. Bouzoubaâ, Selfconsolidating concrete incorporating new viscosity modifying admixtures, Cem. Concr. Res. 34 (6) (2004) 917-926.

[4] M. Sonebi, Rheological properties of grouts with viscosity modifying agents as diutan gum and welan gum incorporating pulverised fly ash, Cem. Concr. Res. 36 (9) (2006) 16091618.

[5] H. Paiva, L.M. Silva, J.A. Labrincha, V.M. Ferreira, Effects of a water-retaining agent on the rheological behaviour of a single-coat render mortar, Cem. Concr. Res. 36 (7) (2006) $1257-1262$.

[6] K.H. Khayat, Viscosity-enhancing admixtures for cement-based materials - an overview, Cem. Concr. Compos. 20 (2-3) (1998) 171-188.

[7] C.F. Ferraris, K.H. Obla, R. Hill, The influence of mineral admixtures on the rheology of cement paste and concrete, Cem. Concr. Res. 31 (2) (2001) 245-255.

[8] M.P. Seabra, H. Paiva, J.A. Labrincha, V.M. Ferreira. Admixtures effect on fresh state properties of aerial lime based mortars. Const. Build. Mater., 23 (2) (2009) 1147-1153.

[9] M.P. Seabra, J.A. Labrincha, V.M. Ferreira. Rheological behaviour of hydraulic limebased mortars. J. Eur. Ceram. Soc., 27 (2-3) (2007). 
[10] H. Paiva, L.P. Esteves, P.B. Cachim, V.M. Ferreira. Rheology and hardened properties of single-coat render mortars with different types of water retaining agents. Const. Build. Mater., $23(2)(2009)$

[11] L. Patural, P. Marchal, A. Govin, P. Grosseau, B. Ruot, O. Devès, Cellulose ethers influence on water retention and consistency in cement-based mortars, Cem. Concr. Res. 41 (1) (2011) 46-55.

[12] L.-M. Zhang, T. Kong, Aqueous polysaccharide blends based on hydroxypropyl guar gum and carboxymethyl cellulose: synergistic viscosity and thixotropic properties, Colloid Polym. Sci. 285 (2) (2006) 145-151.

[13] R. Bouras, A. Kaci, M. Chaouche, Influence of viscosity modifying admixtures on the rheological behavior of cement and mortar pastes, Korea-Aust. Rheol. J. 24 (1) (2012) 35-44. [14] A. Leemann, F. Winnefeld, The effect of viscosity modifying agents on mortar and concrete, Cem. Concr. Compos. 29 (5) (2007) 341-349.

[15] C. Brumaud, R. Baumann, M. Schmitz, M. Radler, N. Roussel, Cellulose ethers and yield stress of cement pastes, Cem. Concr. Res. 55 (2014) 14-21.

[16] L. Bertrand, S. Maximilien, R. Guyonnet, Wedge Splitting Test: a test to measure the polysaccharide influence on adhesion of mortar on its substrate, Proceedings of the $11^{\text {th }}$ International Congress on Polymers in Concrete, Berlin, 2004, pp. 569-576.

[17] A. Jenni, L. Holzer, R. Zurbriggen, M. Herwegh, Influence of polymers on microstructure and adhesive strength of cementitious tile adhesive mortars. Cem. Concr. Res. 35 (1) (2005) 35-50.

[18] J. Pourchez, B. Ruot, J. Debayle, E. Pourchez, P. Grosseau, Some aspects of cellulose ethers influence on water transport and porous structure of cement-based materials, Cem. Concr. Res. 40 (2) (2010) 242-252. 
[19] B. Biasotti, M. Giudici, V. Langella, U Pfeiffer, Highly substituted hydroxypropylguar: a strong contribution to construction chemistry, Proceedings of the $3^{\text {rd }}$ International Dry mix Mortar Conference, Nürnberg, 2011, pp. 2-9.

[20] T. Poinot, A. Govin, P. Grosseau, Impact of hydroxypropylguars on the early age hydration of Portland cement, Cem. Concr. Res. 44 (2013) 69-76.

[21] T. Poinot, A. Govin, P. Grosseau, Importance of coil-overlapping for the effectiveness of hydroxypropylguars as water retention agent in cement-based mortars, Cem. Concr. Res. 56 (2014) 61-68.

[22] T. Poinot, A. Govin, P. Grosseau, Influence of hydroxypropylguars on rheological behavior of cement-based mortars, Cem. Concr. Res. 58 (2014) 161-168.

[23] M. Cappellari, A. Daubresse, M. Chaouche, Influence of organic thickening admixtures on the rheological properties of mortars: Relationship with water-retention, Constr. Build. Mater. 38 (2013) 950-961.

[24] D. Risica, A. Barbetta, L. Vischetti, C. Cametti, M. Dentini, Rheological properties of guar and its methyl, hydroxypropyl and hydroxypropyl-methyl derivatives in semidilute and concentrated aqueous solutions, Polymer. 51 (9) (2010) 1972-1982.

[25] R. Lapasin, L. De Lorenzi, S. Pricl, G. Torriano, Flow properties of hydroxypropyl guar gum and its long-chain hydrophobic derivatives, Carbohydr Polym. 28 (3) (1995) 195-202. [26] J. Plank, Applications of biopolymers and other biotechnological products in building materials, Appl. Microbiol. Biotechnol. 66 (1) (2004) 1-9.

[27] Standard EN 197-1, Cement - Part 1: Composition, specifications and conformity criteria for common cements, 2012. 
[28] T. Poinot, K. Benyahia, A. Govin, T. Jeanmaire, P. Grosseau, Use of ultrasonic degradation to study the molecular weight influence of polymeric admixtures for mortars, Constr. Build. Mater. 47 (2013) 1046-1052.

[29] Standard EN 196-1, Methods of testing cement - Part 1: Determination of strength, 2006. [30] Standard C1506-09, Standard test Method for Water Retention of Hydraulic CementBased Mortars and Plasters. ASTM. (2009).

[31] NF DTU 26.1, Travaux d'enduits de mortiers, (2008).

[32] H.A. Barnes, Q.D. Nguyen, Rotating vane rheometry - a review, J. Non-Newton. Fluid. 98 (1) (2001) 1-14.

[33] A. Ait-Kadi, P. Marchal, L. Choplin, A.S. Chrissemant, M. Bousmina, Quantitative analysis of mixer-type rheometers using the Couette analogy, Can. J. Chem. Eng. 80 (6) (2002) 1166-1174.

[34] T.H. Phan, M. Chaouche, M. Moranville, Influence of organic admixtures on the rheological behaviour of cement pastes, Cem. Concr. Res. 36 (10) (2006) 1807-1813.

[35] N. Roussel, G. Ovarlez, S. Garrault, C. Brumaud, The origins of thixotropy of fresh cement pastes, Cem. Concr. Res. 42 (1) (2012) 148-157.

[36] W.M. Herschel, R. Bulkley, Measurements of consistency as applied to rubber-benzene solutions, Am. Soc. Test. Mater. Proc. 26 (1926) 621-633.

[37] T. Poinot, M.-C. Bartholin, A. Govin, P. Grosseau, Influence of the polysaccharide addition method on the properties of fresh mortars, Cem. Concr. Res. 70 (2015) 50-59.

[38] J. Wang, P. Somasundaran, D.R. Nagaraj, Adsorption mechanism of guar gum at solidliquid interfaces, Miner. Eng. 18 (1) (2005) 77-81. 
[39] H. Lombois-Burger, P. Colombet, J.L. Halary, H. Van Damme, On the frictional contribution to the viscosity of cement and silica pastes in the presence of adsorbing and non adsorbing polymer, Cem. Concr. Res. 38 (2008) 1306-1314.

[40] V.N. Kislenko, Polymer Adsorption at Solid Surface, in Tóth J. (Ed.), Adsorption: Theory, Modeling, and Analysis, Dekker, Ed. New York, (2002) 743-801.

[41] Y. Cheng, K.M. Brown, R.K. Prud'homme, Characterization and Intermolecular Interactions of Hydroxypropyl Guar Solutions, Biomacromolecules. 3(3) (2002) 456-461.

[42] S. Simon, J. Dugast, D. Le Cerf, L. Picton, G. Muller, Amphiphilic polysaccharides. "Evidence for a competition between intra and intermolecular associations in dilute system, Polymer. 44 (26) (2003) 7917-7924.

[43] R.J. Flatt, Y.F. Houst, A simplified view on chemical effects perturbing the action of superplasticizers, Cem. Concr. Res. 31 (2001) 1169-1176.

[44] X. Ma, M. Pawlik, Adsorption of guar gum onto quartz from dilute mixed electrolyte solutions, Journal of Colloid and Interface Science. 298 (2006) 609-614.

[45] D. Bülichen, J. Kainz, J. Plank, Working mechanism of methyl hydroxyethyl cellulose (MHEC) as water retention agent, Cem. Concr. Res. 42 (7) (2012) 953-959.

[46] C. Marliere, E. Mabrouk, M. Lamblet, P. Coussot, How water retention in porous media with cellulose ethers works, Cem. Concr. Res. 42 (11) (2012) 1501-1512.

[47] E. Volpert, J. Selb, F. Candau, Influence of the Hydrophobe Structure on Composition, Microstructure, and Rheology in Associating Polyacrylamides Prepared by Micellar Copolymerization, Macromolecules. 29 (1996) 1452-1463

[48] A.N. Semenov, J.-F. Joanny, A.R. Khokhlov, Associating polymers: equilibrium and linear viscoelasticity, Macromolecules. 28 (1995) 1066-1075. 
[49] M. Collepardi, Self-compacting concrete: what is new? Proceedings of the $7^{\text {th }}$ CANMET/ACI Conference on Superplasticizer and Other Chemical Admixtures in Concrete, Berlin, Germany, 2003, pp. 1-16.

[50] M. Palacios, R.J. Flatt, F. Puertas, A. Sanchez-Herencia, Compatibility between Polycarboxylate and Viscosity-Modifying Admixtures in Cement Pastes, Proceedings of the $10^{\text {th }}$ International Conference on Superplasticizers and Other Chemical Admixtures in Concrete, Prague, 2012, pp. 29-42. 